\title{
ATIVIDADE EPILÉPTICA FOCAL DURANTE SONO INDUZIDO NA SINDROME DE LENNOX-GASTAUT SECUNDÁRIA
}

\author{
J. G. SPECIALI* - L. A. L. RESENDE ***
}

RESUMO -- Quinze pacientes com síndrome de Lennox-Gastaut secundária foram submetidos a exames clinicos e eletrencefalográficos mensais por períodos de tempo que variaram de 1 a 9 anos. Os exames eletrencefalográficos foram realizados durante sono induzido por barbitúrico. Doze pacientes apresentaram atividade epiléptica focal. No presente trabalho descrevemos a localização, morfologia e freqüéncia dos paroxismos epilépticos focais.

\section{Fucal epileptic activity during induced sleep in the secoudary lenmox-Gastaut syndrome.}

SUMMARY - Fifteen patients with secondary Lennox-Gastaut syndrome were submitted to monthly clinical and electroencephalographic examinations for periods of time varying from 1 to 9 years. Twelve of these patients showed focal epileptic activity during induced sleep. In the present report we describe the localization, morphology and frequency of these focal discharges.

Atividade epiléptica focal na sindrome de Lennox-Gastaut tem sido relatada entre 10 e $15 \%$ dos casos 1,2 , mas sem descrições detalhadas. No presente trabalho descrevemos a localização, morfologia e freqüência dos paroxismos epilépticos focais durante sono induzido em crianças com sindrome de Lennox-Gastaut secundária.

\section{MATERIAL E METODOS}

Quinze pacientes con sindrome de Lennox-Gastaut foram selecionados entre 68 pacientes sequidos no Ambulatório de Neurologia do Hospital das Clínicas da FMRP. A síndrome foi caracterizadi de acordo com critérios clínicos e eletrencefalográficos (EEG) estabelecidos yor Gastaut e col. (3). Foram utilizados os seguintes critérios de seleção: sindrome de Lennox-Gastaut secundária; ocorréncia de pelo menos uma crise epiléptica por dia a despeito do tratamento cínico; idade de inicio da sindrome inferior a 7 anos; ausência de sindrome de West prévia; EEG realizados durante sono induzido como parte do seguimento clínico-laboratorial.

Us pacientes foram examinados mensalmente por períodos de tempo que variaram de 1 a 9 anos. Em cada avaliação, foram submetidos a EEG durante fases II e III de sono induzido por secobarbital sódico, em aparelho Grass modelo VI de 8 canais. Os eletrodos foram colocados de acordo com o sistema internacional 10-20. Utilizamos impedância sempre inferior a $5 \mathrm{~K} \Omega$, sensibilidade de $7 \mathrm{~mm} / 50 \mathrm{uV}$, constante de tempo $=0,3$ fijtros de 30 e $60 \mathrm{~Hz}$, velocidade do papel de $3 \mathrm{~cm} / \mathrm{seg}$.

Departamento de Neuro-Psiquiatria e Psicologia Médica, Faculdade de Medicina de Ribeirão Preto (FMRP), Universidade de São Paulo: * Professor Livre-Docente; ** Mestre em Neurologia, Auxiliar de Ensino do Departamento de Neurologia e Psiquiatria, Faculdade de Medicina de Botucatu, Universidade Estadual de São Paulo (UNESF). 


\section{RESULTADOS}

Dos 15 pacientes estudados, três não apresentaram paroxismos epilépticos focais. Na tabela 1 mostramos as freqüências dos vários tipos de paroxismos focais. Onze pacientee mostraram mais que um tipo de paroxismo durante o seguimetto. Espiculas e ondas agudas foram os achados mais comuns. Na tabela 2 são apresentadas as localizaçōes dos diferentes tipos de paroxismos: na vertical está indjcado o número de pacientes com determinada localizaçāo de paroxismos epilépticos focais; na horizontal indicamos o número de pacientes com determinado tipo de paroxismo epiléptico focal (a soma na vertical ou na horizontal nảo pode ser feita, pois um determinado paciente pode apresentar diferentes tipos de paroxismos, ou diferentes localizaçōes). Atividade epiléptica focal foi detectada principalmente nas regióes central e parjeto-temporal. Houve mudanca na localização dos paroxismos de um exume para outro, ou, em contrapartida, duas ou três localizacōes foram detectadas no mesmo EEG. Atividade delta localizada foi considerada epiléptica devido ao elevado potencial, ondas delta com aspecto de ondas agudas e ocorrência de espiculas ou ondas agudas na mesma projeção. Consideramos complexos do tipo ponta-onda lentos, aqueles com frequtências inferiores a $2.5 \mathrm{nz}$; complexos ponta-onda rápidos, aqueles com frequị̂ncias superiores : $3,5 \mathrm{~Hz}$ e complexos ponta-onda, aqueles com freqüências de 2,5 a $3,5 \mathrm{~Hz}$.

\begin{tabular}{|c|c|c|c|}
\hline & Tipo de paroxismo & N' de casos & $\%$ \\
\hline & Espícula & 11 & 73 \\
\hline & Onda aguda & 7 & 46 \\
\hline & Ponta-onda (lento) & 5 & 33 \\
\hline & Onda delta & 4 & 26 \\
\hline & Ponta-onda (rápido) & 2 & 13 \\
\hline & Ponta-onda & 2 & 13 \\
\hline & Sem atividade epiléptica focal & 3 & 20 \\
\hline & Total & 15 & 100 \\
\hline
\end{tabular}

Tabela 1 - Tipos de atividade epiléptica focal detectudos dentre 15 pucientes com sindrome de Lennox-Gastrut secundaria.

\begin{tabular}{|c|c|c|c|c|c|c|}
\hline \multirow[t]{2}{*}{ 1.ccalizaçāo } & \multicolumn{4}{|c|}{ Tipo de paroxismo } & \multirow[b]{2}{*}{$\begin{array}{c}\text { Ponta-onda } \\
\text { (rápido) }\end{array}$} & \multirow[b]{2}{*}{ Ponta-onda } \\
\hline & Espícula & Onda acruda & $\begin{array}{c}\text { Ponta-onda } \\
\text { (lento) }\end{array}$ & Onda delta & & \\
\hline Central & 8 & 3 & 1 & 2 & - & 1 \\
\hline Parieto-temporal & 2 & 2 & 4 & 2 & 1 & 1 \\
\hline Frontai & 4 & 2 & - & - & - & $\cdot-$ \\
\hline Temporal & 5 & 4 & - & - & - & ... \\
\hline Parietal & 3 & 1 & 一 & - & 1 & - \\
\hline Occipital & 1 & 3 & - & - & -- & 一 \\
\hline Parassagital & 1 & 1 & - & 1 & - & - \\
\hline
\end{tabular}

Tabela 2 - Localizaçós dos diferentes tipos de atividade epiléftica focal.

\section{COMENTARIOS}

Atividade epiléptica focal na sindrome de Lennox-Gastaut tem sido relatada na literatura, mas não detalhadamente. Gastaut e col. ${ }^{3}$ descreveram um "foco temporal bem definido" que precede, acompanha ou sucede a fase dos complexos difusos tipo ponta-onda lentos. Blume e col.1 constataram espículas focais em 7 pacientes e multifocais em 8, correspondendo aproximadamente a $10 \%$ dos casos de sindrome de Lennox-Gastaut estudados. Markand 6 , dentre 83 pacientes, encontrou espículas focais em $14(16 \%)$ e espiculas multifocais independentes em $5(6 \%)$. 
A alta freqüência de paroxismos epilépticos focais que detectamos pode ser explicada por: muitos exames foram feitos em cada paciente, ao contrário de apenas dois ou três obtidos por outros investigadores; metodologia utilizada, pois estudamos todas as atividades epilépticas focais que surgiram durante seguimento, enquanto outros, como Blume e col 1 , estudaram paroxismos focais apenas no primeiro exame; o fato de que selecionamos os casos mais graves, com impossibilidade de controle das crises epilépticas; a realizaçāo de nossos exames durante sono, que ativa as espiculas focais 4,5 .

Não encontramos relatos de regiōes com maior incidência de atividade epiléptica focal na sindrome de Lennox-Gastaut. Nosso estudo mostra que as regiôes mais frequentemente envolvidas sāo: central, parieto-temporal, temporal, parietal, occipital e parasagital, em ordem decrescente de freqüência (Tabela 2). Dentre as atividades epilépticas registradas em nossos pacientes, a mais freqüente foi espícula, com polaridade sempre negativa e curta duração (25-50 milisegundos). Atividade epiléptica focal do tipo ponta-onda de frequêencias variadas ocorreu menos frequentemente que espiculas e ondas agudas. Complexos do tipo ponta-onda foram sempre irregulares, mesmo aqueles de $3 \mathrm{~Hz}$. Atividade epiléptica focal do tipo delta estava associada a espiculas ou ondas agudas na mesma projeção.

Observamos que diferentes tipos de paroxismos ocorreram preferencialmente em determinadas regiōes: espiculas na regiāo central, complexos tipo ponta-onda na região parieto-temporal. Ondas agudas se distribuiram uniformemente (Tabela 2). Atividade epiléptica focal nāo constituiu achado constante, surgindo durante certos períodos da evolução e desaparecendo durante outros. Em exames sucessivos e, algumas vezes durante um mesmo exame, ocorreram mudanças de localizaçăo dos paroxismos epilépticos focais. As caracteristicas das atividades epilépticas focais registradas em nossos pacientes foram similares àquelas da "epilepsia com focos intercríticos múltiplos" 7,8.

Nosso trabalho foi conduzido no sentido de demonstrar que a síndrome de Lennox-Gastaut secundária deve ser considerada como forma de epilepsia multifocal secundariamente generalizada.

\section{REFERENCIAS}

1. Blume WT, David RB, Gomez MR - Generalized sharp and slow wave complexes: associated clinical features and longterm follow-up. Brain 96:289, 1973.

2. Degen R, Degen HE - Das schlaf-EEG Patienten mit mioclonischastatischen Anfällen. Z EEG-EMG 14:106, 1983.

3. Gastaut H, Roger J, Soulayrol R, Tassinari CA, Régis H, Dravet C — Childhood epileptic encephalopathy with diffuse slow-waves (otherwise know as «petit mal variant») or Lennox-syndrome, Epilepsia $7: 139,1966$.

4. Karbowski K, Vassela F, Schneider H - Electroencephalographic aspects of Lennox syndrome. Eur Neurol 4:301, 1970.

5. Loiseau $P$, Cohadon $\mathbf{S}$ - Les épilepsies à foyers EEG intercritiques multiples. Rev EEG Neurophysiol $11: 259,1981$.

6. Markand ON - Slow spike-wave activity ir EMG and associated clinical features: often called «Lennox» or «Lennox-Gastaut» syndrome. Neurology $27: 746,1977$.

7. Melo AN - Contribuição ao Estudo da Epilepsia com Descargas Epileptiformes Multifocais Independentes na Infância. Tese. Faculdade de Medicina, Ribeirão Preto, 1983.

8. Noriega-Sanchez A, Markand ON - Clinical and electroencephalographic correlations of independent multifocal spike discharges. Neurology 26:667, 1976. 